\title{
1-(3,4,5-Trimethoxyphenyl)ethane-1,2-diyl Esters, a Novel Compound Class with Potent Chemoreversal Activity
}

\author{
Hsin-Yi Hunga,c, Emika Ohkoshia ${ }^{a}$, Masuo Goto ${ }^{a}$, Kyoko Nakagawa-Goto ${ }^{a, b}$, and Kuo-Hsiung \\ Lee $^{\mathrm{a}, \mathrm{c},{ }^{*}}$ \\ aNatural Products Research Laboratories, UNC Eshelman School of Pharmacy, University of \\ North Carolina, Chapel Hill, North Carolina 27599-7568, USA \\ bDivision of Pharmaceutical Sciences, Graduate School of Natural Science and Technology, \\ Kanazawa University, Kakuma-machi, Kanazawa 920-1192, Japan \\ ${ }^{\circ}$ Chinese Medicine Research and Development Center, China Medical University Hospital, \\ Taichung, Taiwan, 401, R.O.C
}

\begin{abstract}
1-(3,4,5-Trimethoxyphenyl)ethane-1,2-diyl esters, which share a fragment from ( \pm )- $3^{\prime}-O-4^{\prime}-O$ bis(3,4-dimethoxycinnamoyl)-cis-khellactone (DMDCK) and $3^{\prime} R, 4^{\prime} R$-disubstituted-2' $2^{\prime} 2^{\prime}$ dimethyldihydropyrano[2,3-f]chromone (DSP), exhibited remarkable chemoreversal activity on multi-drug resistant human nasopharyngeal carcinoma (KB) when combined with three anticancer drugs, paclitaxel, vincristine and doxorubicin. Among 15 novel synthesized analogs, bistrimethoxybenzoyl derivative $\mathbf{1 5}$ was the most active (340-fold more active than verapamil when used with vincristine) followed by two di-cinnamoyl derivatives, $\mathbf{1 0}$ and 11, and then dicyclohexanecarbonyl derivative 9. All aliphatic chain derivatives, 3-5, showed no activity. Structure-activity relationship study indicated that a di-ester structure was critical to enhance the activity resulting from the maintenance of the spatial arrangement proposed by the pharmacophore based on the verapamil-binding site. Further mechanism of action study showed $\mathbf{1 5}$ inhibited mainly P-glycoprotein efflux pump function, while $\mathbf{1 3}$ exhibited an additional multidrug resistance-associated protein efflux pump function.
\end{abstract}

\section{Keywords}

1-(3,4,5-Trimethoxyphenyl)ethane-1,2-diyl esters; Chemoreversal activity; KB cell line

Chemotherapy is an important strategy for cancer treatment. However, it is difficult to eliminate all tumor cells, resulting in relapse with resistant cancer cells. Emerging evidence suggests that epithelial-mesenchymal transition (EMT)-type cells and cancer stem cells (CSCs) or initiating cells are more resistant to conventional chemotherapy and are linked to anticancer drug resistance leading to failure of chemotherapy. ${ }^{1}$ Another issue of chemotherapeutic resistance is the tumor microenvironment, in which cancer cells are surrounded by various sets of non-transformed cells and a heterogenous stromal

\footnotetext{
(C) 2012 Elsevier Ltd. All rights reserved.

*Corresponding author K.H.L.: Phone: 919-962-0066; Fax: 919-966-3893; khlee@ unc.edu.

Publisher's Disclaimer: This is a PDF file of an unedited manuscript that has been accepted for publication. As a service to our customers we are providing this early version of the manuscript. The manuscript will undergo copyediting, typesetting, and review of the resulting proof before it is published in its final citable form. Please note that during the production process errors may be discovered which could affect the content, and all legal disclaimers that apply to the journal pertain.
} 
compartment. $^{2}$ The interaction between tumor cells and normal cells in this tumor microenvironment occurs via secreted and surface-bound proteins, and is critical for tumor progression. $^{2}$

In addition to the tumor microenvironment, cancer cells also adapt other mechanisms to deal with chemotherapeutic agents. ${ }^{3}$ Over-expression of drug efflux transporters such as Pglycoprotein (P-gp) and multidrug resistance-associated proteins (MRPs) is the primary cause leading to multidrug resistance. Numerous studies have explored chemosensitizer development. However, clinical drugs are still unavailable.

Verapamil (VRP), the first generation chemosensitizer, was precluded from clinical use due to its high toxicity, but it is used as a positive control in various chemosensitizing research efforts. Before the structure of P-glycoprotein (P-gp) was resolved, a pharmacophore model based on the verapamil-binding site was proposed. ${ }^{4}$ In this model, the pharmacophore for Pgp substrates or inhibitors consists of two hydrophobic planes, three optional hydrogen bond (HB) acceptor points, and one optional HB donor point spatially arranged like a butterfly shape. ${ }^{4}$ Based on this model, ( \pm )- $3^{\prime}-O-4^{\prime}-O$-bis-(3,4-dimethoxycinnamoyl)-cis-khellactone (DMDCK) was found to be a hit and was also confirmed to exhibit chemosensitizing ability. ${ }^{5}$ Moreover, $3^{\prime} R, 4^{\prime} R$-disubstituted- $2^{\prime}, 2^{\prime}$-dimethyldihydropyrano[2,3- $f$ chromone (DSP) analogs sharing similar structures to DMDCK have also been reported to overcome multidrug resistance (MDR) in an acquired MDR human nasopharyngeal carcinoma (KB) cell line. ${ }^{6}$ With inspiration from the above results, a simplified scaffold, 1-(3,4,5trimethoxyphenyl)ethane-1,2-diyl ester, was designed (Fig. 1) to provide an ethane-1,2-dilyl ester partial structure mimicking a fragment in both DSP and DMDCK. By esterifying different substituents, the importance of the spatial arrangement mentioned in the pharmacophore model can be evaluated. According to results based on another model, P-gp inhibitors should show high hydrophobicity, with two or more aromatic rings, a methoxy group (HB acceptor) on the aromatic ring, and one or two protonatable nitrogens. ${ }^{7}$ Verapamil also contains two methoxy group on an aromatic ring. In addition, previous study of DDB substitutions also showed increasing activity along with an increased number of methoxy substituents. ${ }^{8}$ Therefore, a trimethoxy-substituted aromatic ring was designed as the basic scaffold in this study.

1-(3,4,5-Trimethoxyphenyl)ethane-1,2-diol (1), a common intermediate for all synthesized compounds, was obtained through Wittig reaction of 3,4,5-trimethoxybenzaldehyde following dihydroxylation with osmium tetroxide and $\mathrm{N}$-methylmorpholine $\mathrm{N}$-oxide (Scheme 1). Various acyl groups, including linear alkyl (3-5), unsaturated fatty acid (8-9), cyclic alkyl (6-7), and aromatic (10-15) side chains, were selected for the structure-activity relationship (SAR) study. Both mono- and di-esters were also synthesized by controlling the equivalents of the acyl chloride to investigate the importance of the spatial arrangement and hydrophobicity. Diesterification of diol was achieved with excess acyl chloride under basic conditions to generate the related diesters, $\mathbf{3 , 5 - 7 , 9}$, and $\mathbf{1 0}$. The bulky acyl chlorides, such as butyryl chloride and cyclohexanecarbonyl chloride, were selectively introduced on only the primary alcohol to produce mono-ester derivatives, $\mathbf{4}$ and $\mathbf{8}$. Monobenzoylester $\mathbf{1 3}$ was prepared through the esterification of the primary alcohol using 1.1 eq. of benzoyl chloride, following silylation of the secondary alcohol. Halogenated compounds are abundant among drug candidates. Therefore, iodinated analogs, $\mathbf{2}, \mathbf{1 1}, \mathbf{1 2}, \mathbf{1 4}$, and 15, were also designed and synthesized through iodination of 3,4,5-trimethoxybenzaldehyde following the same reaction sequence as mentioned above (Scheme 1). Newly synthesized derivatives, 1-15 (Table 1), were screened for cytotoxicity and chemoreversal effects on KB and MDR KB cells in the absence (-) or presence (+) of paclitaxel. 
Most of the analogs exhibited no significant cytotoxicity at $10 \mu \mathrm{M}$ against four tumor cell lines, A549, DU145, KB and KBvin (Supplementary Data, Table S1). Reversal activity of 1(3,4,5-trimethoxyphenyl)ethane-1,2-diyl esters using paclitaxel is shown in Table 2 . In the absence of paclitaxel, most compounds had no cytotoxic effect, while chemoreversal effects were seen on KBvin cells but not on KB cells in the presence of paclitaxel. This result indicates that these compounds are specifically toxic for MDR KB cells when combined with the current anticancer drug. Brief SAR correlations were observed as follows. Among the alkyl esters, acetyl (3), butyryl (4) and dodecanoyl (5) groups showed no chemoreversal activity. In the case of short fatty acid esters (6 and 7), moderate reversal effects were observed, indicating a conjugated system may contribute to chemoreversal activity. Interestingly, compound 9 with bulky di-cyclohexanecarbonyl esters displayed remarkable reversal activity, while mono-cyclohexanecarbonyl ester $(\mathbf{8})$ totally lost activity. This fact suggested that di-esters would be better than mono-esters, probably due to higher hydrophobicity, a critical feature for drug efflux pump inhibitors or the importance of the butterfly shape proposed in the pharmacophore model. ${ }^{4}$ The ester derivatives containing an aromatic ring showed relatively better reversal ability than the aliphatic ester derivatives. Especially, cinnamoyl ester 10 and 2-iodo-3,4-dimethoxycinnamoyl ester 11, with the same side chain as DMDCK, totally reversed the cytotoxicity of paclitaxel in the KBvin cell line. Similarly, 2-iodo-mono-3,4,5-trimethoxybenzoyl (14) as well as -di-3,4,5trimethoxybenzoyl (15) esters displayed high chemoreversal effects. These four compounds resulted in $0 \%$ of KBvin cell survival at $10 \mu \mathrm{M}$. The comparison of $\mathbf{1 0}$ and $\mathbf{1 1}$, as well as $\mathbf{1 2}$ and 13, suggested that iodination of the C-2 position is not very important for the activity. In the case of benzoyl ester, similar effects were shown regardless of mono-ester (14) or diester (15). In a comparison of $\mathbf{1 2}$ and $\mathbf{1 4}$, the silyl group led to reduced chemoreversal when an iodo group was present at the $\mathrm{C}-2$ position and also increased cytotoxicity in the presence of paclitaxel. Among the potent derivatives 9-15, compound 12 displayed cytotoxicity. Therefore, only 9-11 and 13-15, mainly cinnamoyl and trimethoxybenzoyl derivatives, were selected for further evaluation with three anticancer drugs, paclitaxel, vincristine and doxorubicin (Table 3). Among the six compounds, trimethoxybenzoyl analogs, 13-15, generally had better chemoreversal ability than cinnamoyl derivatives (10 and 11) when combined with the three anticancer drugs. The reversal activity was similar between $\mathbf{1 0}$ and 11. Among compounds 13-15 with mono- or di-3,4,5-trimethoxylbenzoyl modification, diester 15 was the most active showing 340-fold more potency than verapamil when used with vincristine. Compounds $\mathbf{1 3}$ with a bulky and hydrophobic tert-butyldimethylsilyl group also showed significant activity. Cyclohexanoyl ester 9 displayed less activity than the other five compounds. These results indicated that hydrophobicity, higher electron density on aromatic group, as well as the maintenance of the spatial arrangement are critical for better chemoreversal activity.

All of the active compounds (9-11 and 13-15) were further evaluated for effects on P-gp efflux function in KBvin cells using fluorescent P-gp substrate calcein-AM (Fig. 2). ${ }^{8}$ Cellular accumulation of calcein was observed in the presence of $10 \mu \mathrm{M}$ compounds, demonstrating that the compounds prevent calcein-AM efflux from KBvin cells by inhibiting P-gp. Compound 15 exhibited two-fold more calcein accumulation than verapamil at the same concentration, indicating $\mathbf{1 5}$ is more potent than verapamil at inhibiting P-gp function. Although 13 showed strong reversal activity compared with verapamil, its inhibitory effect on the P-gp efflux pump was relatively weaker than verapamil. Hence, 13 may inhibit other drug transporters, such as multidrug resistance-associated proteins (MRPs). The inhibitory effect of $\mathbf{1 3}$ on MRPs can be determined by using a fluorogenic MRP substrate Fluo-3 AM, which is accumulated in KB cells and pumped out from KBvin cells (Fig. 3). Although no significant Fluo-3 fluororescence was seen in KBvin cells pretreated with 15, cellular accumulation of Fluo-3 was observed in KBvin cells pre-treated 
with 13. These results demonstrate that $\mathbf{1 3}$ inhibits efflux pump function of MRPs, whereas $\mathbf{1 5}$ does not affect MRPs. Taken together, $\mathbf{1 5}$ selectively inhibits P-gp and $\mathbf{1 3}$ can interfere with both P-gp and MRPs.

In this study, we demonstrated that a simplified scaffold containing the core of DMDCK and DSP exhibited excellent chemoreversal activity. Fifteen ester analogs were designed, synthesized, and evaluated for chemoreversal activity. Based on the results, di-ester modification is critical for better activity, indicating the importance of maintaining the spatial arrangement of the compound. SAR study indicates that di-trimethoxylbenzoyl substitution resulted in the most activity, followed by di-3,4-dimethoxylcinnamoyl and then di-cycloheanecarbonyl substitutions. Aliphatic groups, such as acetyl or butyryl, led to no activity. Mechanism of action study showed that these compounds inhibited the drug efflux function of P-gp, which increases the cellular accumulation of anticancer drugs, such as paclitaxel, vincristine, and doxorubicin. Moreover, compound $\mathbf{1 3}$ showed additional inhibitory activity against MRPs. Further modifications and more detailed SAR will be reported in the near future.

\section{Supplementary Material}

Refer to Web version on PubMed Central for supplementary material.

\section{Acknowledgments}

This study was supported by grant CA-17625-32 from the National Cancer Institute, NIH, awarded to K. H. Lee. Support in part was also due to the Cancer Research Center of Excellence (CRC) (DOH-100-TD-C-111-005). We would like to thank Dr. Mitch Eddy (NIEHS/NIH) for providing a fluorescence microscope.

\section{References}

1. Singh A, Settleman J. EMT, cancer stem cells and drug resistance: an emerging axis of evil in the war on cancer. Oncogene. 2010; 29:4741. [PubMed: 20531305]

2. Gilbert LA, Hemann MT. Chemotherapeutic resistance: surviving stressful situations. Cancer Res. 2011; 71:5062. [PubMed: 21771909]

3. Fodale V, Pierobon M, Liotta L, Petricoin E. Mechanism of cell adaptation: when and how do cancer cells develop chemoresistance? Cancer J. 2011; 17:89. [PubMed: 21427552]

4. Pajeva IK, Wiese M. Pharmacophore model of drugs involved in P-glycoprotein multidrug resistance: explanation of structural variety (hypothesis). J. Med. Chem. 2002; 45:5671. [PubMed: 12477351]

5. Fong WF, Shen XL, Globisch C, Wiese M, Chen GY, Zhu GY, Yu ZL, Tse AK, Hu YJ. Methoxylation of 3',4'-aromatic side chains improves P-glycoprotein inhibitory and multidrug resistance reversal activities of 7,8-pyranocoumarin against cancer cells. Bioorg. Med. Chem. 2008; 16:3694. [PubMed: 18313307]

6. Zhou T, Shi Q, Bastow KF, Lee KH. Antitumor agents 286. Design, synthesis, and structure-activity relationships of 3'R,4'R-disubstituted-2',2'-dimethyldihydropyrano[2,3-f]chromone (DSP) analogues as potent chemosensitizers to overcome multidrug resistance. J. Med. Chem. 2010; 53:8700. [PubMed: 21082774]

7. Pajeva IK, Globisch C, Wiese M. Combined pharmacophore modeling, docking, and 3D QSAR studies of ABCB1 and ABCC1 transporter inhibitors. Chem Med Chem. 2009; 4:1883. [PubMed: 19768722]

8. Hung HY, Ohkoshi E, Goto M, Bastow KF, Nakagawa-Goto K, Lee KH. Antitumor Agents 293. Non-toxic dimethyl-4, $4^{\prime}$-dimethoxy-5,6,5', $6^{\prime}$-dimethylenedioxybiphenyl-2,2' -dicarboxylate (DDB) analogs chemosensitize multidrug resistant cancer cells to clinical anticancer drugs. J. Med. Chem. 2012; 14:5413. [PubMed: 22612652] 


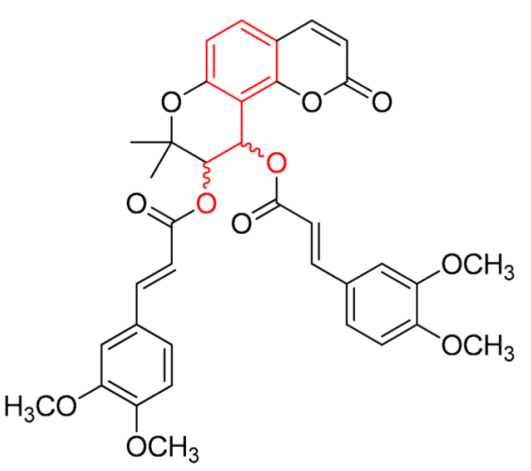<smiles>[R]C1c2c(ccc3c(=O)cc(CC)oc23)OC(C)(C)C1[R]</smiles>

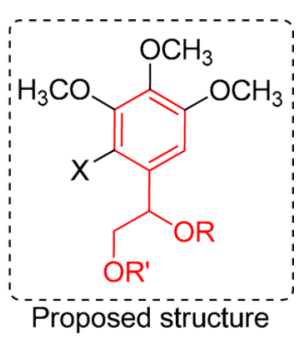

\section{for This Study}

(+/-)DMDCK

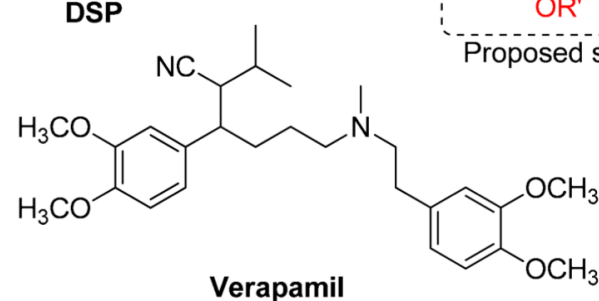

Figure 1. Structures of ( \pm ) DMDCK, DSP, and Verapamil and Proposed Structure for This Study 


\section{cellular calcein accumulation}

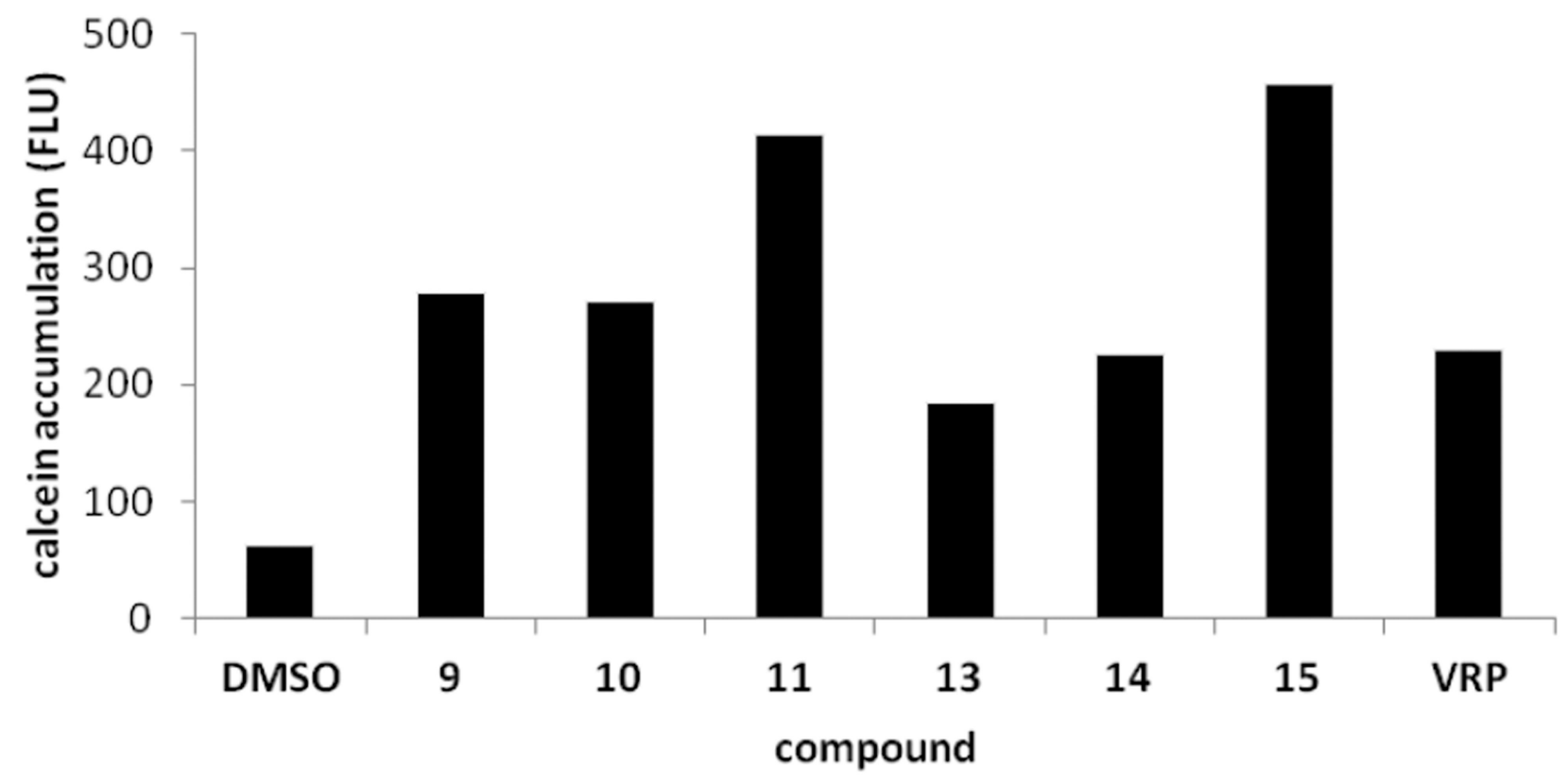

Figure 2. Inhibitory Effects of Compounds on P-gp Function in KBvin Cells

The KBvin cells were pretreated with the compounds $(10 \mu \mathrm{M})$ followed by addition of calcein-AM. The cellular accumulation of calcein is represented by fluorescent units (FLU). Cellular accumulation of calcein demonstrates inhibition of efflux activity of Pgp. Data with mean \pm SD of three independent experiments are shown in suppl. Table S2. 

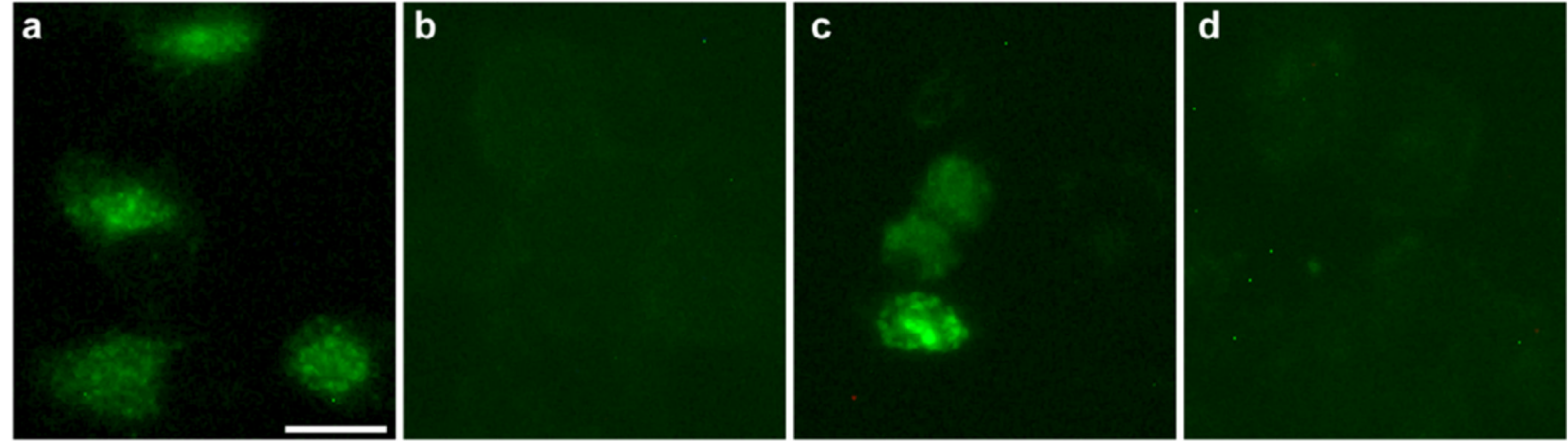

Figure 3. Effect of 13 on MRP Function in KBvin Cells

$\mathrm{KB}$ (a) or KBvin (b, c, d) cells were pre-treated with DMSO (a, b), 13 (c), or $\mathbf{1 5}$ (d) for $1 \mathrm{~h}$ before addition of Fluo-3 AM for $2 \mathrm{~h}$. Cellular accumulation of Fluo-3, a fluorescence MRPs substrate, was observed in KB cells (a), and effluxed from KBvin cells (b). Cellular retention of Fluo-3 fluorescence was observed in KBvin cells treated with $\mathbf{1 3}$ (c) yet undetectable in cells treated with $15(\mathrm{~d})$. (Bar, $50 \mu \mathrm{m}$. 
<smiles>[X]c1c(C=O)cc(OC)c(OC)c1OC</smiles>
$\mathrm{X}=\mathrm{H} \square \mathrm{a}$
$\mathrm{X}=\mathrm{I}$<smiles>[X]c1c(C=C)cc(OC)c(OC)c1OC</smiles>

$\mathrm{X}=\mathrm{H}$ or $\mathrm{I}$<smiles>[X]c1c([C@@H](O)CO)cc(OC)c(OC)c1OC</smiles>

1: $\mathrm{X}=\mathrm{H}$ 2: $X=1$<smiles>[R]OCC([R7])c1cc(OC)c(OC)c(OC)c1[X]</smiles>

3, 5-7, 9, 10: $X=H, R=R^{\prime}=A c y l$ 4, 8: $X=H, R=A c y l, R^{\prime}=H$ 11, 15: $X=I, R=R^{\prime}=$ Acyl $\rightarrow$ 12: $X=I, R=$ TBS, $R^{\prime}=3,4,5$-triOMe-Bz

13: $X=H, R=$ TBS, $R^{\prime}=3,4,5$-triOMe-Bz 14: $X=I, R=H, R^{\prime}=3,4,5$-triOMe-Bz

Reagents and conditions: (a) trifluoroacetate, iodine; (b) $\mathrm{Ph}_{3} \mathrm{PCH}_{3} \mathrm{Br}$, tBuOK, toluene; (c) OsO $\mathrm{O}_{4}, \mathrm{NMO}, \mathrm{THF}, 55^{\circ} \mathrm{C}$;

(d) $\mathrm{RCOCl}, \mathrm{Et}_{3} \mathrm{~N}, \mathrm{CH}_{2} \mathrm{Cl}_{2} ;$ (e) 2,6-lutidine, TBSOTf, $\mathrm{CH}_{2} \mathrm{Cl}_{2},-78{ }^{\circ} \mathrm{C}$

Scheme 1. Synthetic Route to 1-(3,4,5-Trimethoxyphenyl)ethane-1,2-diyl Esters 
Table 1

Structures of 1-(3,4,5-Trimethoxyphenyl)ethane-1,2-diyl Esters.

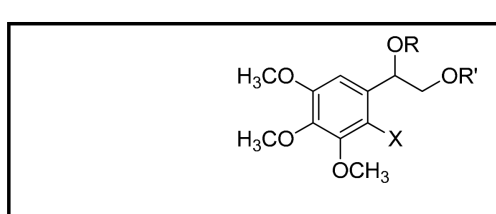

\begin{tabular}{|c|c|c|c|}
\hline No. & X & R & $\mathbf{R}^{\prime}$ \\
\hline $\mathbf{1}$ & H & H & H \\
\hline $\mathbf{2}$ & I & H & H \\
\hline 3 & H & O N & O \\
\hline
\end{tabular}

$$
\begin{array}{l|l|}
\hline 4 & \mathrm{H}
\end{array}
$$$$
\text { N }
$$

\begin{tabular}{|c|c|c|c|}
\hline 4 & $\mathrm{H}$ & $\mathrm{H}$ & \\
\hline $\mathbf{5}$ & $\mathrm{H}$ & $\mathrm{O}$ & $\mathrm{O}$ \\
\hline
\end{tabular}

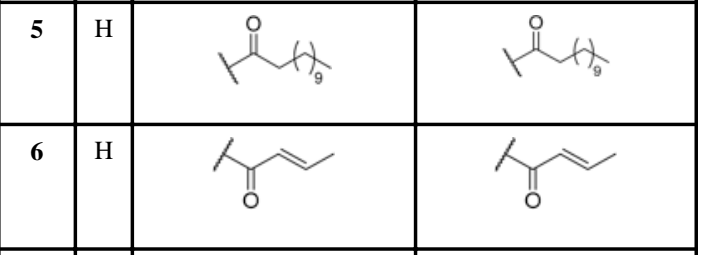

7 (4)

8

$$
\mathrm{H}
$$

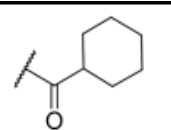

\begin{tabular}{l|l|l}
9 & $\mathrm{H}$ \\
\hline
\end{tabular}
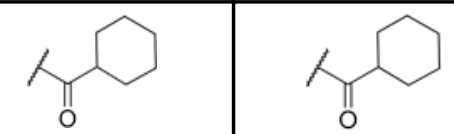

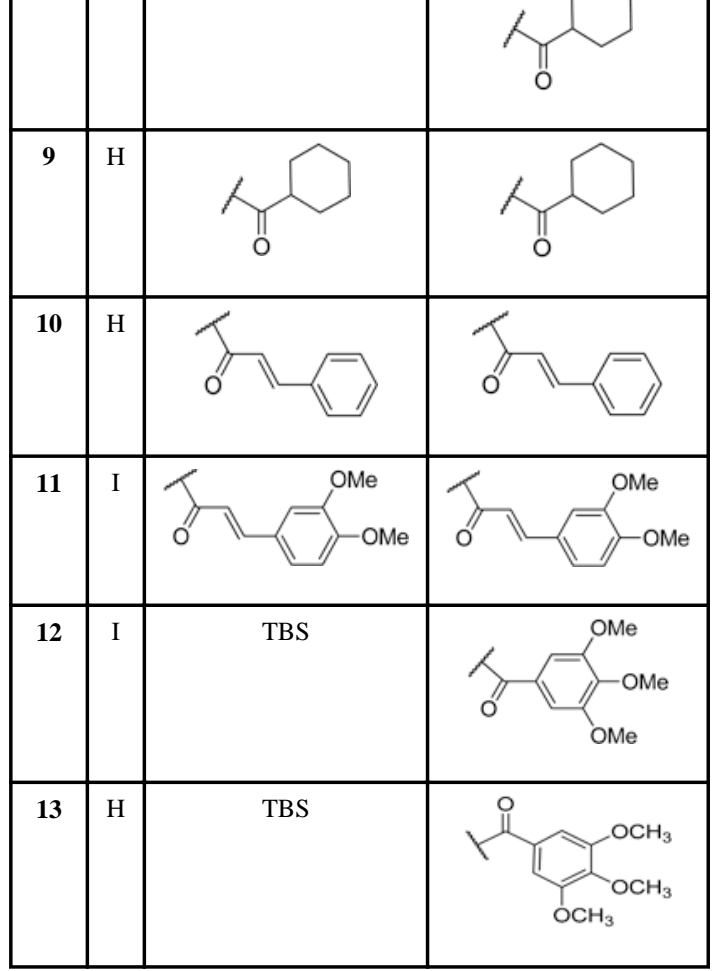

Bioorg Med Chem Lett. Author manuscript; available in PMC 2013 December 15. 


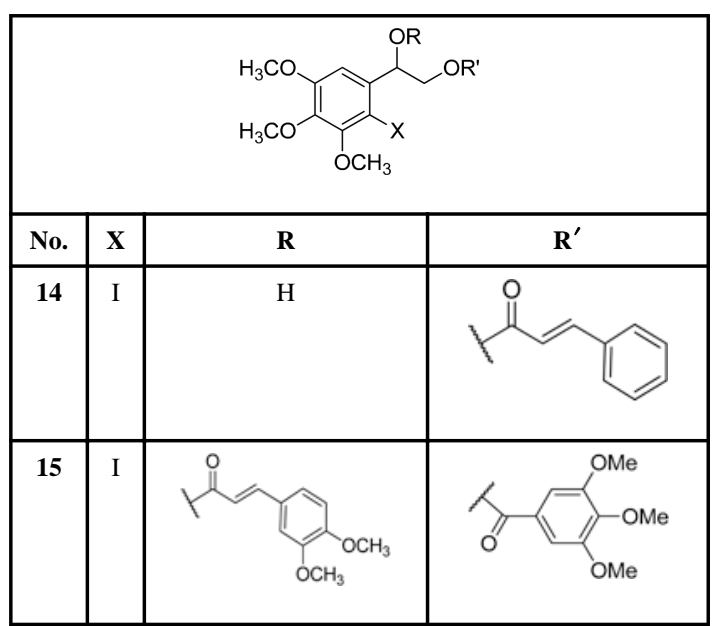




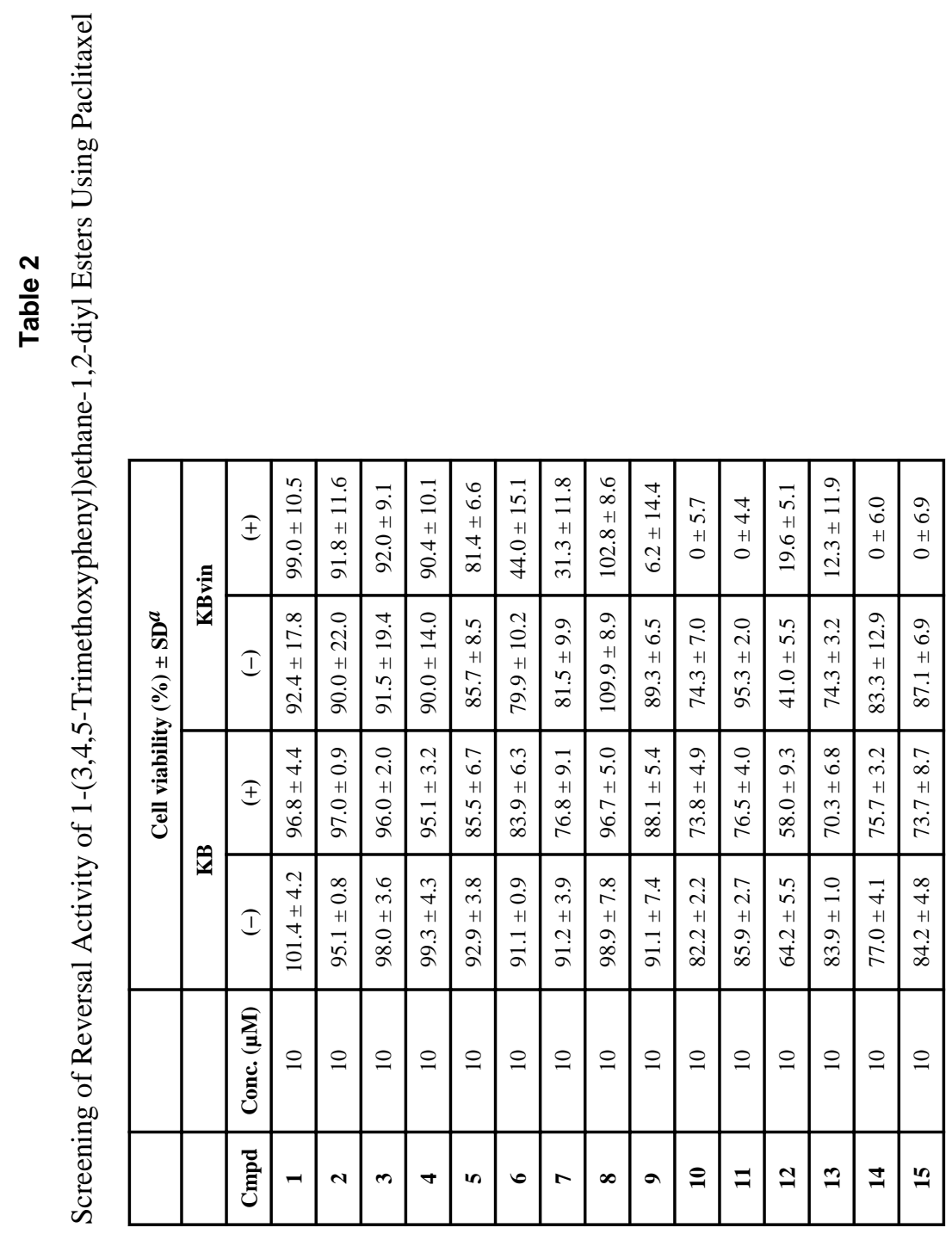


